\title{
The FDA and PLATO investigators death lists: Call for a match
}

\author{
Victor Serebruany ${ }^{1}$, Jean-Francois Tanguay ${ }^{2}$, and Thomas Marciniak ${ }^{3}$ \\ ${ }^{1}$ Johns Hopkins University \\ ${ }^{2}$ Montreal Heart Institute \\ ${ }^{3}$ Retired from FDA
}

January 11, 2021

\begin{abstract}
Purpose: The FDA-issued PLATO trial dataset revealed that some primary deaths causes (PDC) were inaccurately reported favoring ticagrelor. However, the PLATO Investigators operated the shorter death list of uncertain quality. We compared if PDC match when trial fatalities were reported to the FDA and by the PLATO investigators. Method: The FDA list contains precisely detailed 938 PLATO deaths, while shorter investigators dataset consists of 905 deaths. We matched 4 vascular (sudden, post-MI, heart failure and stroke), and 3 non-vascular (cancer, sepsis and suicide) PDC between death lists. Results: There were more sudden deaths in the shorter list than in the FDA dataset (161 vs.138; p <0.03), post-AMI (373 vs.178; p $<0.001)$ but fewer heart failure deaths (73 vs.109; $\mathrm{p}=0.02)$. Stroke numbers match well (39 vs. $37 ; \mathrm{p}=\mathrm{NS})$ with only 2 ticagrelor cases removed. Cancer matched well (32 vs.31; $\mathrm{p}=\mathrm{NS}$ ), and sepsis cases were identical (30 vs. 30; P=NS). However, 2 extra clopidogrel suicides in the shorter list are impossible to comprehend. Conclusions: The PLATO trial PDC were mismatched between FDA and Investigators sets. We are kindly asking the ticagrelor sponsor or/and concerned PLATO Investigators to clarify the PDC dataset match.
\end{abstract}

\section{PERSPECTIVE}

\section{CARDIOVASCULAR DISEASE}

The FDA and PLATO investigators death lists:

\section{Call for a match}

Victor L. Serebruany, ${ }^{1}$ Jean-Francois Tanguay, ${ }^{2}$ and Thomas A. Marciniak ${ }^{3}$

${ }^{1}$ Johns Hopkins University, Department of Neurology, Stroke Unit, Baltimore, Maryland, USA;

${ }^{2}$ Montreal Heart Institute, Université de Montréal, Montreal, Quebec, Canada;

3. Physician, Bethany Beach, Delaware, USA

Running title: Reporting deaths in PLATO trial

Total words: 1,214

Correspondence: Victor Serebruany, MD, PhD, Division of Neurology, Johns Hopkins University School of Medicine, 14110 Rover Mill Road, West Friendship, MD, 21794, USA (vserebr1@jhmi.edu; heartdrug@aol.com ).

\section{Abstract}


Purpose: The FDA-issued PLATO trial dataset revealed that some primary deaths causes (PDC) were inaccurately reported favoring ticagrelor. However, the PLATO Investigators operated the shorter death list of uncertain quality. We compared if PDC match when trial fatalities were reported to the FDA and by the PLATO investigators.

Method : The FDA list contains precisely detailed 938 PLATO deaths, while shorter investigators dataset consists of 905 deaths. We matched 4 vascular (sudden, post-MI, heart failure and stroke), and 3 non-vascular (cancer, sepsis and suicide) PDC between death lists.

Results: There were more sudden deaths in the shorter list than in the FDA dataset (161 vs.138; $<<0.03$ ), post-AMI (373 vs.178; $\mathrm{p}<0.001)$ but fewer heart failure deaths (73 vs.109; $\mathrm{p}=0.02)$. Stroke numbers match well (39 vs. $37 ; \mathrm{p}=\mathrm{NS}$ ) with only 2 ticagrelor cases removed. Cancer matched well (32 vs.31; $\mathrm{p}=\mathrm{NS}$ ), and sepsis cases were identical (30 vs. $30 ; \mathrm{P}=\mathrm{NS}$ ). However, 2 extra clopidogrel suicides in the shorter list are impossible to comprehend.

Conclusions: The PLATO trial PDC were mismatched between FDA and Investigators sets. We are kindly asking the ticagrelor sponsor or/and concerned PLATO Investigators to clarify the PDC dataset match.

Key Words Clinical trial, ticagrelor, clopidogrel, cause of death, mortality

\section{BACKGROUND}

Ticagrelor is an oral, reversible, direct-acting inhibitor of the adenosine diphosphate receptor $\mathrm{P}_{2} \mathrm{Y} 12$ that was tested against clopidogrel in the PLATO trial. Remarkably for the oral antiplatelet agents, PLATO Investigators reported that ticagrelor reduced death from vascular causes $(4.0 \%$ vs. $5.1 \%, \mathrm{P}=0.001)$ and death from any cause $(4.5 \%$, vs. $5.9 \% \mathrm{P}<0.001) .{ }^{1}$ Such a strong mortality benefit was surprising because of opposite death counts in the Phase II DISPERSE studies, the mismatch between reasonable myocardial infarction and very high death rates in PLATO, the inverse US outcomes, the unusually delayed timing of benefit, the last minute change of trial governance from the TIMI Group to the sponsor, the late introduction of electronic clinical research forms (eCRF), ${ }^{2}$ and some striking "errors" revealed by the FDA, including for example, a clopidogrel patient who was reported dead but later experienced non-fatal bleeding. ${ }^{3}$ However, until now, no hard evidence of misreported death records with definite proof was publicly available. As of today, ticagrelor holds a superiority recommendation over clopidogrel for acute coronary syndromes in European, ${ }^{4}$ Canadian, ${ }^{5}$ and American ${ }^{6}$ guidelines based mostly on the results of the PLATO trial. ${ }^{1}$

\section{PLATO DEATHS LISTS}

Lately our Task Force gained access to the detailed dataset of 938 PLATO deaths reported to the FDA. We matched those records with local patient-level data from sites controlled by the sponsor, and found that the existence, precise dates and proper causes of some deaths in PLATO were inaccurately reported. ${ }^{7}$ Several clopidogrel deaths were reported earlier than actual, while their causes were switched from "non-vascular" or "unknown" to "vascular". In contrast, some ticagrelor deaths were reported later while other vascular deaths were incorrectly entered in the FDA list as "non-vascular" or "unknown". ${ }^{7}$ We explore here any differences between the FDA-issued death list $(\mathrm{n}=938)$ and the shorter PLATO Investigators dataset $(\mathrm{n}=905)$. The overall numbers of fatalities and some primary causes for both lists are presented in the Table.

With regard to the discrepancy in total PLATO deaths, it is our understanding that the dataset we have in possession includes deaths after the analysis period that are not counted in the primary efficacy analysis, but the absolute majority of deaths (over 900) should match precisely (Figure). Indeed, we identified several events when the patient never received a single drug dose, serious enrollment mistakes, and inclusion/exclusion errors, but the most common restriction was the length of follow-up. In fact, the PLATO death count was restricted to no more than 12 months follow-up duration. Any death within the analysis period should be counted, although PLATO also excluded deaths after "withdrawal of consent" or "volunteer discontinuations mostly for ticagrelor patients. ${ }^{3,8}$ Importantly, there were some PLATO patients who were still on drug and died 12-16 months after enrollment while the trial was still active in the second half of 2008, but these patients were excluded as being beyond the analysis timeframe. Finally, we are skeptical that the 
reductions in the FDA-issued list removed more ticagrelor $(n=21)$ than clopidogrel $(n=12)$ events for the final count of 905 deaths used in all PLATO publications and affiliated presentations.

Furthermore, we presumed that the precise death characteristics and single primary causes of all remaining PLATO deceased patients should be identical between the FDA and trial investigators. All 905 deceased patients in the "official" trial dataset should be included in the broader list submitted to the FDA. Since some of the 938 deaths were reported inaccurately to the FDA, we started looking at which of the erroneous records were transferred into the smaller revised PLATO pool used in the original ${ }^{1}$ and over 90 peer-reviewed secondary publications. Examining details of 2 high-quality secondary PLATO publications ${ }^{9,10}$ focused on mortality revealed several fundamental problems outlined in the Table.

The well-structured and detailed data from the PLATO Investigators' clearly indicate that Tables 5 (vascular) and 6 (non-vascular) reported primary causes of deaths. ${ }^{9}$ The FDA list also identify a single primary cause of death, perhaps "unknown" or "other" but just one cause. However, looking at the PLATO Investigators' statements reveals obvious discrepancies. With regard to reported numbers of vascular deaths the significant differences were observed between FDA- and trial investigators lists. There were more sudden deaths in the shorter list than in the FDA dataset (161 vs.138; $\mathrm{p}<0.03$ ), post-AMI (373 vs.178; $<<0.001)$ but fewer heart failure deaths ( 73 vs.109; $\mathrm{p}=0.02$ ). Stroke numbers match well (39 vs. $37 ; \mathrm{p}=\mathrm{NS}$ ) with only 2 ticagrelor cases removed. Among non-vascular causes of deaths cancer matched well (32 vs.31; $\mathrm{p}=\mathrm{NS}$ ), and sepsis cases were identical (30 vs. $30 ; \mathrm{P}=\mathrm{NS}$ ). However, suicides deserve special attention. Two extra cases ${ }^{9}$ in the shorter list are impossible to comprehend. Not only suicide (PLATO code 12-5) is a valid primary death cause, but both "missing" patients are from the clopidogrel arm. We directly approached PLATO Investigators with the detailed specific inquiry regarding these 2 suicides no reported to the FDA but got no response or any explanation regarding the 2 extra deaths in the Investigators' list. Not solving such a simple issue raises questions regarding not only vascular deaths prevention but also the claimed all-cause mortality benefit of ticagrelor.

\section{IMPRESSION}

As of today, the evidence suggests that the FDA-issued and Investigators datasets for PLATO deaths were different and do not match. Because the FDA appears to have erroneously judged and approved ticagrelor based on inaccurate death reports, the main message of this paper is to call for a match. Importantly, 905 deaths reported in almost 100 of papers in top journals by PLATO Investigators should be identical with the FDA report. However, it seems that primary death causes, especially vascular ones, were misreported or flawed in some way independent from the FDA submission. Moreover, the death classification codes applied were different as well and it is unclear why such efforts were not synchronized with the FDA. The current scenario looks like parallel reality. One inaccurate death list is provided to the FDA for gaining regulatory approval, while another different dataset with even more favorable ticagrelor benefit is issued to trial investigators that may have led to multiple false-positive reports. We are kindly asking the ticagrelor sponsor or/and concerned PLATO Investigators to help us with the deaths dataset clarification.

Acknowledgements: Our true appreciation to clinical and statistical FDA reviewers on ticagrelor New Drug Application 22-433 for their integrity and courage with the "no approval" recommendation. At the time of NDA 22-433 review, the senior author of this paper was a Medical Team Leader for the FDA cardiorenal division. These findings reflect our own knowledge, based on Task Force analyses, and are not the opinions of Johns Hopkins University or Montreal Heart Institute. Drs. Serebruany and Marciniak but not

Dr. Tanguay had full access to the FDA-issued PLATO death list.

Conflicts of Interest: None.

\section{REFERENCES}

1. Wallentin L, Becker RC, Budaj, et al. Ticagrelor versus Clopidogrel in Patients with Acute Coronary Syndromes. N Engl J Med 2009; 61:1045-1057 
2. Serebruany VL. Paradoxical excess mortality in the PLATO trial should be independently verified.Thromb Haemost 2011;105:752-759.

3. The FDA ticagrelor review of complete response. Available for download at: http://www.accessdata.fda.gov/drugsatfda_docs/nda/2011/022433Orig1s000MedR.pdf Assessed June 15, 2020

4. Neumann F-J, Sousa-Uva M, Ahlsson A, et al. ESC/EACTS Guidelines on myocardial revascularization. Eur Heart $J$ 2019; 40: 87-165

5. Mehta SR, Bainey KR, Cantor WJ, et al. 2018 Canadian Cardiovascular Society/Canadian Association of Interventional Cardiology Focused Update of the Guidelines for the Use of Antiplatelet Therapy. Can J Cardiol 2018;34:214-233

6. Levine GN, Bates ER, Bittle JA. et al. ACC/AHA Guideline Focused Update on Duration of Dual Antiplatelet Therapy in Patients With Coronary Artery Disease. JACC; 2016; 68:1082-1115

7. Serebruany V, Tanguay J-F, Benavides MA, et al. Verifying Death Reports in the Platelet Inhibition and Patient Outcomes (PLATO) Trial.Amer J Therap 2020; 27:e563-e572

8. DiNicolantonio JJ, Tomek A. Misrepresentation of vital status follow-up: challenging the integrity of the PLATO trial and the claimed mortality benefit of ticagrelor versus clopidogrel. Int $J$ Cardiol 2013;169:145-146

9. Varenhorst C, Alström U, Braun OO, et al. Causes of mortality with ticagrelor compared with clopidogrel in acute coronary syndromes. Heart 2014;100:1762-1769

10. Storey RF, James SK, Siegbahn A, et al. Lower mortality following pulmonary adverse events and sepsis with ticagrelor compared to clopidogrel in the PLATO study. Platelets 2014;25:517-525

Table. Primary deaths causes in the FDA- and PLATO Investigators datasets

\begin{tabular}{|c|c|c|c|c|c|}
\hline Death Cause & PLATO Code & $\begin{array}{l}\text { FDA-C } \\
(n=518)\end{array}$ & $\begin{array}{l}\text { FDA-T } \\
(n=420)\end{array}$ & $\begin{array}{l}\text { Investigators- } \\
C \\
(n=506)\end{array}$ & $\begin{array}{l}\text { Investigators- } \\
\mathbf{T} \\
(\mathbf{n}=399)\end{array}$ \\
\hline Vascular: & $11-1$ 11-2 11-5 & $78901862 \quad 17$ & 6088214714 & 981941842 & 631791931 \\
\hline Sudden & $11-1012-3$ & 123 & 17 & 17323 & 1517 \\
\hline Myocardial & $12-512-8$ & & & & \\
\hline Infarction & & & & & \\
\hline Stroke Heart & & & & & \\
\hline failure Non- & & & & & \\
\hline vascular: & & & & & \\
\hline Cancer Suicide & & & & & \\
\hline Sepsis & & & & & \\
\hline
\end{tabular}

C- clopidogrel; T - ticagrelor;

Figure. Anticipated PLATO deaths count algorithm transition from the FDA to Investigators 